\title{
Long-term survival after intrahepatic cholestasis of pregnancy: a follow-up of 571 mothers
}

\author{
Suvi-Tuulia Hämäläinen ${ }^{1,2,3}$ M.D.
}

Kaisa Turunen ${ }^{1}$ M.D., Ph.D.

Kari J Mattila ${ }^{1}$ M.D., Ph.D.

Elise Kosunen ${ }^{1,3}$ M.D., Ph.D.

\section{Markku Sumanen ${ }^{1}$ M.D., Ph.D.}

1 Department of General Practice, Faculty of Medicine and Health Technology, Tampere University, Arvo Ylpön katu 34, 33520 Tampere, Finland

2 Janakkala Health Centre, Tapailanpiha 13 B, 14200 Turenki, Finland

3 Science Center, Tampere University Hospital, 33521 Tampere, Finland

4 Centre for General Practice, Pirkanmaa Hospital District, 33521 Tampere, Finland

Correspondence and requests for reprints to: Suvi-Tuulia Hämäläinen, MD, general

practitioner, Janakkala Health Centre, Tapailanpiha 13 B, 14200 Turenki, Finland

Email: suvi.hamalainen@tuni.fi Telephone: +358 368011 Fax: +358 36801209 


\begin{abstract}
OBJECTIVE: Intrahepatic cholestasis of pregnancy (ICP) is the most common liver disorder during pregnancy. ICP has been associated with morbidity but little is known about women's long-term survival. Our aim was to determine whether ICP is associated with mothers' long-term survival.
\end{abstract}

STUDY DESIGN: The study population comprised 571 women with ICP in at least one pregnancy seen at Tampere University Hospital in Finland between 1969-1988. The reference group comprised 1333 women: the previous and the following participant in the maternity ward diary. The data were obtained from Statistics Finland in March 2017 containing deaths among the study participants between 1971-2015. The follow-up time of the cohort was 27-46 years. The Kaplan-Meier method was used.

RESULTS: Totally, 39 of the mothers with ICP (6.8\%) and 111 of the reference group (8.3\%) had died by the end of $2015(p=0.267)$. The mean survival time of ICP women was 77.4 years and of the reference group 79.2 years $(p=0.288)$. The mean survival time from labour in the ICP group was 45.0 years and in the reference group 44.8 years $(p=0.259)$.

CONCLUSIONS: Based on this study ICP does not seem to be associated with women's survival. There is no need to follow-up ICP mothers' health because of the nonexistent risk of premature death.

\title{
Keywords
}

intrahepatic cholestasis of pregnancy, death, mortality, survival, women's health issues 


\section{Introduction}

Intrahepatic cholestasis of pregnancy (ICP) is a pregnancy-specific liver condition which occurs most often in the late second or third trimester. The incidence is estimated to be between $0.2 \%$ and $2 \%$ and it varies geographically $(1,2)$. The disease is more common in South Asia, South America and Scandinavia. The diagnosis requires the key symptom pruritus and a rise in serum bile acids and transaminases (1). During pregnancy the importance of ICP is related to its effects on the fetus (3). Fetal complications such as prematurity, fetal distress and stillbirth are associated with ICP $(4,5)$. Within 48 hours of delivery the mother's ICP symptoms usually resolve, and biochemical abnormalities resolve within 2-8 weeks (6).

Genes, hormonal and environmental factors have an effect on the pathogenesis of intrahepatic cholestasis of pregnancy. Several gene mutations have an impact on its pathogenesis (7-14). Family clustering, the presence of ethnic and geographic variations, and mutations in gene coding for hepatobiliary transport proteins refer to genetic predisposition of ICP $(1,2)$. Progesterone and estrogen seem to have an effect on the disease, and ICP patients have an altered steroidogenesis (15). Selenium deficiency and time of year may be involved in ICP's pathogenesis (16-18).

Recently, there has been growing interest in comorbidity associated with intrahepatic cholestasis of pregnancy. Increased risk for hepatobiliary diseases and cancers, some 
autoimmune diseases, and cardiovascular diseases have been found (19-21). Women who have experienced ICP seem to have more gestational diabetes and pre-eclampsia than controls $(22,23)$. The disease seems to be linked to hepatitis C infection $(20,24-26)$. ICP women was found to have a higher HCV viral load (26).

Little attention has been paid to ICP women's survival. The condition being the most common pregnancy related liver disease, it is important to know about ICP's association regarding survival and public health. According to the writers' knowledge, ICP mothers' survival over such a long follow-up time has not been studied prior to this study. As ICP is associated with specific genetic profile and morbidity, the aim of this study was to determine whether there is an association between ICP and survival.

\section{Material and methods}

The study population comprised all ICP pregnancies at Tampere University Hospital, Finland, between 1969 and 1988. Patients with ICP were identified in the hospital discharge register using diagnosis codes. From 1969 to 1986, ICD-8 was used at Tampere University Hospital. Because ICD-8 did not include a precise code for ICP, we checked all the obstetric codes that might contain ICP: 637.9 Toxicosis NUD, 639.00 Pruritus, 639.01 Icterus gravis, 639.09 Necrosis acuta et subacuta hepatis, and 639.98 Aliae definitae. Thereafter, we checked the written diagnosis behind the code, and if it referred to ICP, we included the case for further selection. ICD-9 was used between 1987 and 1988, and it contained the appropriate codes 6467A Hepatosis gravidarum and 6467X 
Hepatopathia alia. The diagnosis was verified from each patient record with the presence of the main symptom of itching and abnormal laboratory test results. At least one of the following was required: ASAT $>35 \mathrm{U} / 1$, ALAT $>40 \mathrm{U} / 1$, or bile acids $6 \mu \mathrm{mol} / 1$ or more. The study population comprised 687 ICP deliveries (27). The diagnosis of ICP was made in the special health care of University Hospital.

The study population included some women with repeated ICP deliveries, and each of these women was studied as an individual case. The ICP group thus contained 575 women. The reference group consisted of two women for each ICP delivery; the previous and the next women in the maternity ward diary. It was verified from the patient records that the women in the reference group were not diagnosed with ICP. Altogether, the reference group consisted of 1374 women. Four women were ruled out from the ICP cases because of a missing personal identity code, as were 41 women from the reference group. The final cohort comprised 571 women with ICP and 1333 women as a reference. Totally five hepatitis cases were diagnosed among the cohort until 2013, and two of them were hepatitis-C.

We collected the cohort's dates of death from Statistics Finland. The data contain all deaths to 2015. The follow-up time of the cohort was 27-46 years. The data were analysed using SPSS for Windows, version 22.0. The results were analysed with the Kaplan-Meier method. 
The patients' consent was not required for this registry study. The study was approved by the Regional Ethics Committee of Tampere University Hospital (R02149) and the National Institute for Health and Welfare in Finland (THL/1051/5.05.00/2014).

\section{Results}

Totally, 39 of the mothers with ICP (6.8\%) and 111 of the reference group (8.3\%) had died by the end of $2015(p=0.267)$. There were no differences between the groups regarding age at labour, at death and age of those who were living at the end of 2015

\section{(Table 1).}

The mean survival time from birth among ICP women was 77.4 years and among reference group 79.2 years, p-value being 0.288 (Figure 1). The mean survival time after labour in the ICP group was 45.0 years and in the reference group 44.8 years, p-value being 0.259 (Figure 2).

\section{Discussion}

Statistically significant differences were not found in the survival between the ICP and the reference groups.

The cohort includes all ICP cases detected in Tampere University Hospital during 1969-1988. Practically 100 percent of the deaths can be found in the data of Statistics Finland (28). The strength of our study is the long follow-up time, 27-46 years after delivery. 
A weakness of the study is that it presents deaths at relatively early ages. Even a longer follow-up time is needed to examine whether ICP is associated with differences in survival among those who die older. The levels of bile acids and/or transaminases used for diagnosis of ICP were somewhat lower than more recent criteria and this can be regarded as a weakness of the study. The lack of knowledge of potential confounding factors such as body mass index can be considered weakness of the study.

Previously ICP has been associated with age over 35 years (29). However, such an association was not found among this cohort. Thus, the cohort can be considered comparable with reference group regarding age. According to a questionnaire study with an excellent response rate, the groups were comparable regarding body mass index and education level (30). In the cohort, ICP women seem to have had fewer deliveries compared to the reference group (31).

Within the same cohort, a questionnaire study showed that smoking was less common among the ICP patients than in the reference group (32). No differences regarding recent alcohol consumption or physical activity were found. Within the same cohort, the women with a history of ICP reported less high cholesterol and high blood pressure requiring medication (30). However, aforementioned diseases and health behaviour had no effect on survival. 
Comorbidity has been recognized to be associated with ICP. In the questionnaire study made within the same cohort, ICP women reported more hypothyroidism and hepatobiliary diseases (30). Hepatobiliary diseases have shown to be associated with ICP in registry-based studies $(19,20)$. Additionally, a registry-based study linked hypothyroidism and some other autoimmune diseases to ICP and found also an increased risk for cardiovascular diseases among ICP women (21). Moreover, gestational diabetes and pre-eclampsia have been associated with ICP $(22,23)$.

The association between intrahepatic cholestasis of pregnancy and cancers has been studied. The questionnaire study suggested an association between ICP and breast cancer (30). In later registry-based studies, the linkage was not found (21,33). Instead, a connection between ICP and hepatobiliary cancer has been found (21). As a summary, ICP increases the woman's risk for certain diseases. Although ICP is associated with certain comorbidity, the survival seems to be similar compared to controls.

Hepatitis C has been associated with increased risk of occurrence of coronary atherosclerosis (34). Although hepatitis C has been associated with ICP $(20,24,25)$, in our cohort there were no differences in the hepatitis $\mathrm{C}$ incidence between ICP women and the reference group. Thus, in our cohort hepatitis C comorbidity does not have an effect on survival. 
ICP is associated with a certain genetic profile $(1,2)$. The same profile may have an influence on the survival among ICP patients. Indeed, ICP itself might not have an effect on the survival but this specific genetic profile might have. This research brings a new insight for long-term aspects of ICP as studying survival over such a long follow-up time.

During the follow-up time no statistically significant differences in the survival between the ICP and the reference group were found. Based on this study ICP does not seem to be associated with women's survival in middle or younger age. This finding is relieving for women with a history of ICP as it is not associated with their life expectation. In addition, there is no need for the health care system to follow-up ICP mothers' health because of the nonexistent risk of premature death.

\section{Conflicts of interest}

None.

\section{Funding}

Tampere University Hospital District's Centre for General Practice funded the payments to the Statistics Finland. This study was partly supported by the Competitive State Research Financing of the Expert Responsibility Area of Tampere University Hospital. 


\section{References}

(1) Williamson C, Geenes V. Intrahepatic cholestasis of pregnancy. Obstetrics \& Gynecology 2014;124:120-133.

(2) Ozkan S, Ceylan Y, Ozkan OV, Yildirim S. Review of a challenging clinical issue: Intrahepatic cholestasis of pregnancy. World J of Gastroenterol 2015;21:7134-7141.

(3) Joshi D, James A, Quaglia A, Westbrook RH, Heneghan MA. Liver disease in pregnancy. Lancet 2010;375:594-605.

(4) Glantz A, Marschall HU, Mattsson LA. Intrahepatic cholestasis of pregnancy: Relationships between bile acid levels and fetal complication rates. Hepatology 2004;40:467-474.

(5) Kawakita T, Parikh LI, Ramsey PS et al. Predictors of adverse neonatal outcomes in intrahepatic cholestasis of pregnancy. Am J Obstet Gynecol 2015;213:570.e1-570.e8.

(6) Geenes V, Williamson C. Intrahepatic cholestasis of pregnancy. World J Gastroenterol 2009;15:2049-2066.

(7) de Vree JM, Jacquemin E, Sturm E et al. Mutations in the MDR3 gene cause progressive familial intrahepatic cholestasis. Proc Natl Acad Sci U S A 1998;95:282-287.

(8) Jacquemin E, Cresteil D, Manouvrier S, Boute O, Hadchouel M. Heterozygous nonsense mutation of the MDR3 gene in familial intrahepatic cholestasis of pregnancy. Lancet 1999;353:210-211.

(9) Dixon PH, Weerasekera N, Linton KJ et al. Heterozygous MDR3 missense mutation associated with intrahepatic cholestasis of pregnancy: evidence for a defect in protein trafficking. Hum Mol Genet 2000;9:1209-1217.

(10) Pauli-Magnus C, Lang T, Meier Y et al. Sequence analysis of bile salt export pump (ABCB11) and multidrug resistance p-glycoprotein 3 (ABCB4, MDR3) in patients with intrahepatic cholestasis of pregnancy. Pharmacogenetics 2004;14:91-102.

(11) Dixon PH, van Mil SW, Chambers J et al. Contribution of variant alleles of ABCB11 to susceptibility to intrahepatic cholestasis of pregnancy. Gut 2009;58:537-544.

(12) Anzivino C, Odoardi MR, Meschiari E et al. ABCB4 and ABCB11 mutations in intrahepatic cholestasis of pregnancy in an Italian population. Dig Liver Dis 2013;45:226-232. 
(13) Dixon PH, Wadsworth CA, Chambers J et al. A comprehensive analysis of common genetic variation around six candidate loci for intrahepatic cholestasis of pregnancy. Am J Gastroenterol 2014;109:76-84.

(14) Dixon PH, Sambrotta M, Chambers J et al. An expanded role for heterozygous mutations of ABCB4, ABCB11, ATP8B1, ABCC2 and TJP2 in intrahepatic cholestasis of pregnancy. Sci Rep 2017;7:11823-017-11626-x.

(15) Parizek A, Hill M, Duskova M et al. A Comprehensive Evaluation of Steroid Metabolism in Women with Intrahepatic Cholestasis of Pregnancy. PloS one 2016;11:e0159203.

(16) Berg B, Helm G, Petersohn L, Tryding N. Cholestasis of pregnancy. Clinical and laboratory studies. Acta Obstet Gynecol Scand 1986;65:107-113.

(17) Kauppila,A, Korpela H, Mäkilä UM, Yrjänheikki E. Low serum selenium concentration and glutathione peroxidase activity in intrahepatic cholestasis of pregnancy. British medical journal 1987;294:150-152.

(18) Reyes H, Baez ME, Gonzalez MC et al. Selenium, zinc and copper plasma levels in intrahepatic cholestasis of pregnancy, in normal pregnancies and in healthy individuals, in Chile. J Hepatol 2000;32:542-549.

(19) Ropponen A, Sund R, Riikonen S, Ylikorkala O, Aittomäki K. Intrahepatic cholestasis of pregnancy as an indicator of liver and biliary diseases: a population-based study. Hepatology 2006;43:723-728.

(20) Marschall HU, Wikström Shemer E, Ludvigsson JF, Stephansson O. Intrahepatic cholestasis of pregnancy and associated hepatobiliary disease: a population-based cohort study. Hepatology 2013;58:1385-1391.

(21) Wikström Shemer E, Stephansson O, Thuresson M, Thorsell M, Ludvigsson J, Marschall H. Intrahepatic cholestasis of pregnancy and cancer, immune-mediated and cardiovascular diseases: A population-based cohort study. J Hepatol 2015;63:456-461.

(22) Wikström Shemer E, Marschall HU, Ludvigsson JF, Stephansson O. Intrahepatic cholestasis of pregnancy and associated adverse pregnancy and fetal outcomes: a 12-year population-based cohort study. BJOG 2013;120:717-723.

(23) Marathe JA, Lim WH, Metz MP, Scheil W, Dekker GA, Hague WM. A retrospective cohort review of intrahepatic cholestasis of pregnancy in a South Australian population. Eur J Obstet Gynecol Reprod Biol 2017;218:33-38.

(24) Locatelli A, Roncaglia N, Arreghini A, Bellini P, Vergani P, Ghidini A. Hepatitis C virus infection is associated with a higher incidence of cholestasis of pregnancy. $\mathrm{Br} \mathrm{J}$ Obstet Gynaecol 1999;106:498-500. 
(25) Paternoster DM, Fabris F, Palu G et al. Intra-hepatic cholestasis of pregnancy in hepatitis C virus infection. Acta Obstet Gynecol Scand 2002;81:99-103.

(26) Belay T, Woldegiorgis H, Gress T, Rayyan Y. Intrahepatic cholestasis of pregnancy with concomitant hepatitis C virus infection, Joan C. Edwards SOM, Marshall University. European journal of gastroenterology \& hepatology 2015;27:372-374.

(27) Turunen K, Sumanen M, Haukilahti RL, Kirkinen P, Mattila K. Good pregnancy outcome despite intrahepatic cholestasis. Scand J Prim Health Care 2010;28:102-107.

(28) Official statistics of Finland. Causes of death [e-publication]. Quality Description: Causes of death 2013. [referred: 15.3.2019]. Helsinki: Statistics Finland 2013.

(29) Heinonen S, Kirkinen P. Pregnancy outcome with intrahepatic cholestasis. Obstet Gynecol 1999;94:189-193.

(30) Turunen K, Mölsä A, Helander K, Sumanen M, Mattila KJ. Health history after intrahepatic cholestasis of pregnancy. Acta Obstet Gynecol Scand 2012;91:679-685.

(31) Mölsä A, Turunen K, Mattila KJ, Sumanen M. Unnecessary confusion about family planning after intrahepatic cholestasis of pregnancy. Contraception 2012;86:639-644.

(32) Turunen K, Helander K, Mattila K, Sumanen M. Health behavior after intrahepatic cholestasis of pregnancy. Health 2013;5:96-101.

(33) Hämäläinen S, Turunen K, Mattila K, Kosunen E, Sumanen M. Intrahepatic Cholestasis of Pregnancy and Cancer: A Cohort Study. Family Medicine \& Medical Science Research 2017;6:216.

(34) Olubamwo OO, Aregbesola AO, Miettola J, Kauhanen J, Tuomainen TP. Hepatitis $\mathrm{C}$ and risk of coronary atherosclerosis - A systematic review. Public Health 2016;138:1225. 
Table 1. Median ages and age ranges of the cohort at labour, at death, and those still living at the end of 2015.

\begin{tabular}{lrccrrrr}
\hline & \multicolumn{3}{c}{ Mothers with ICP } & & \multicolumn{3}{c}{ Reference group } \\
\cline { 2 - 4 } \cline { 6 - 8 } & $\mathrm{n}$ & median age & age range & & $\mathrm{n}$ & median age & age range \\
\hline At labour & 571 & 27.6 & $16.9-41.1$ & & 1333 & 27.1 & $15.0-46.4$ \\
At death & 39 & 56.7 & $34.1-78.5$ & & 111 & 55.9 & $28.1-83.8$ \\
Living at the end of 2015 & 532 & 63.7 & $48.0-80.8$ & & 1222 & 63.3 & $46.3-85.0$ \\
\hline
\end{tabular}




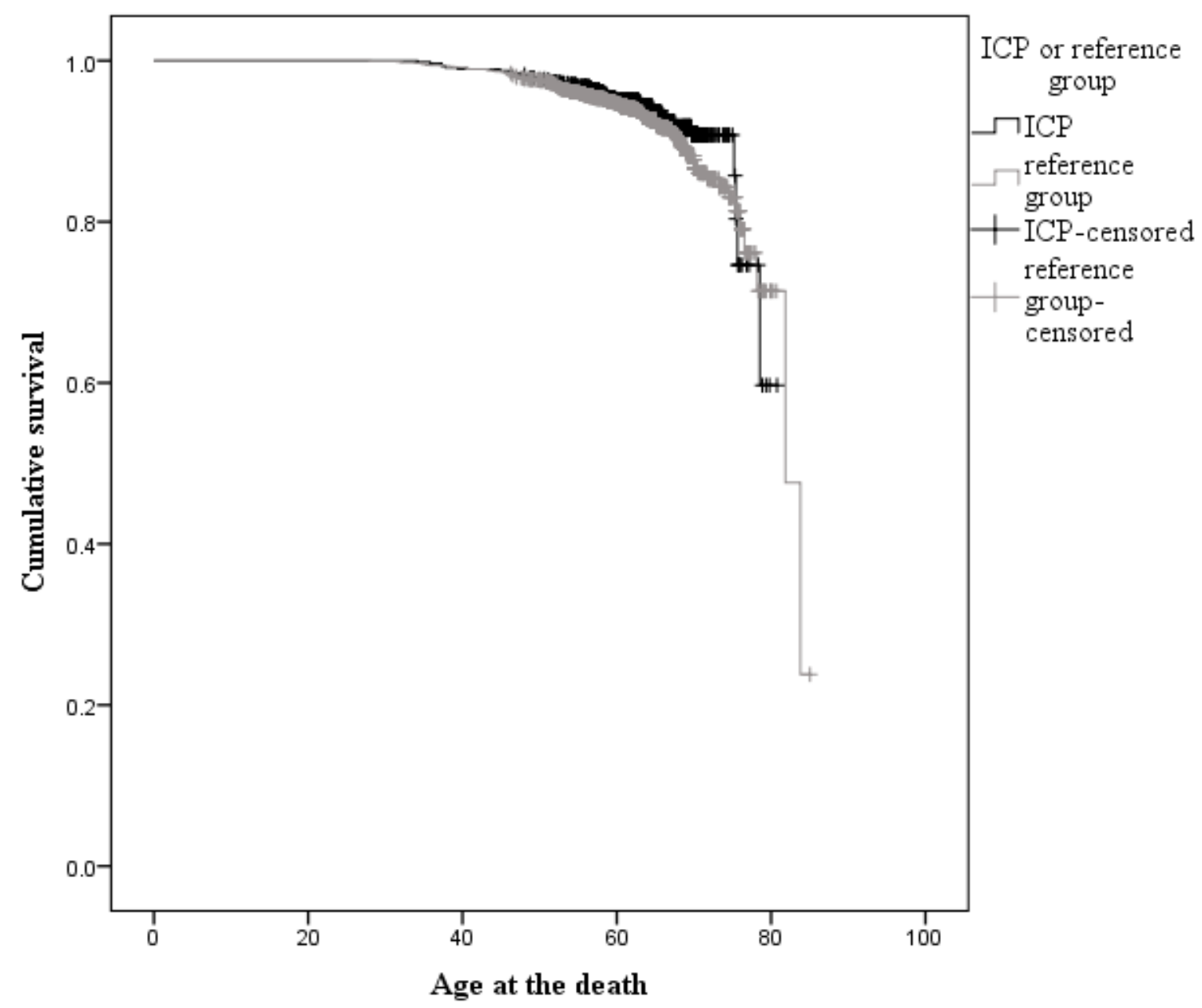

Figure 1. Survival in the ICP and the control groups regarding age. The censored line indicates the subjects who are alive at the end of the follow-up time. 


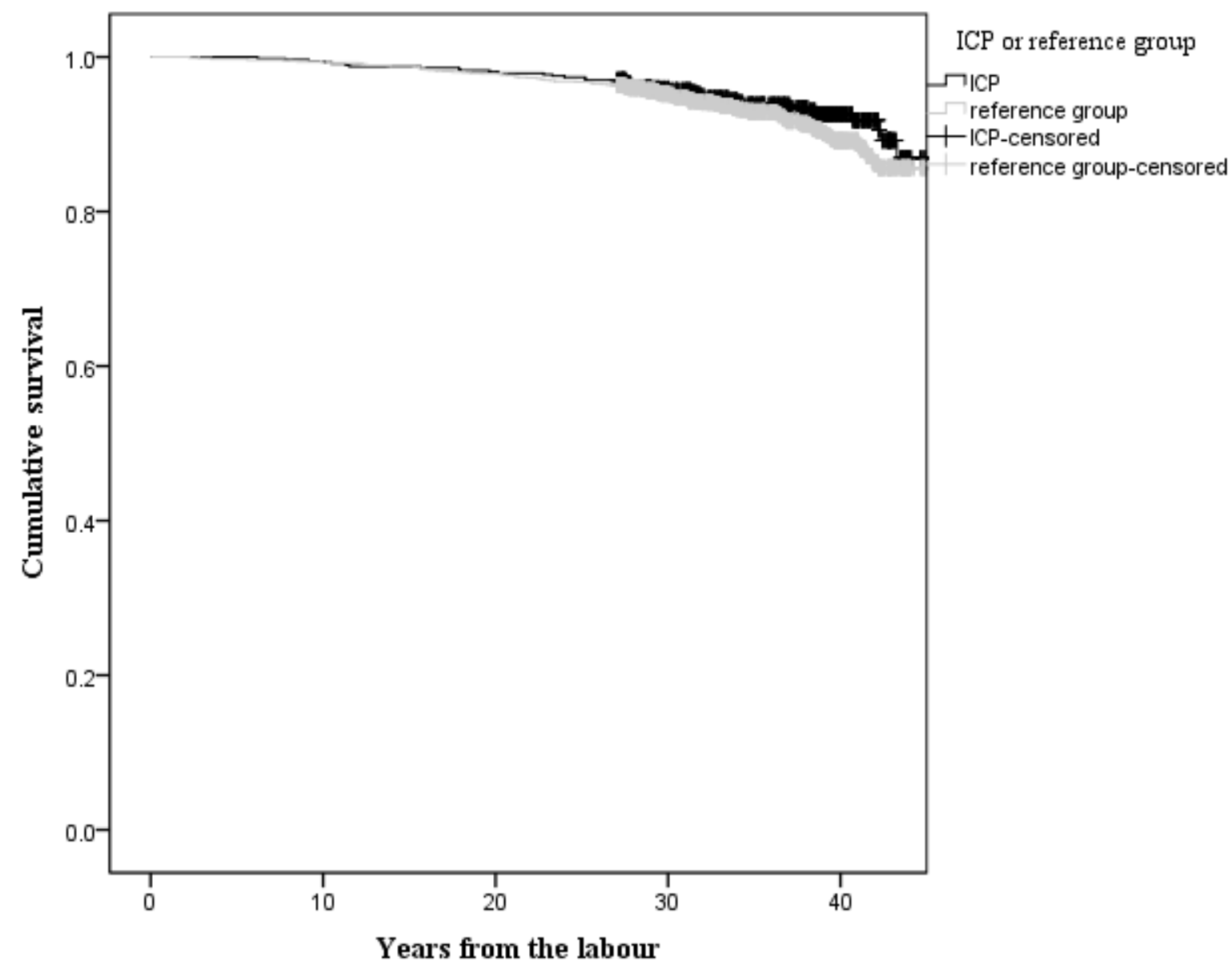

Figure 2. Survival in the ICP and the control groups regarding years after labour. The censored line indicates the subjects who are alive at the end of the follow-up time. 